ARTICLE

\title{
Ultralong lifetime and efficient room temperature phosphorescent carbon dots through multi- confinement structure design
}

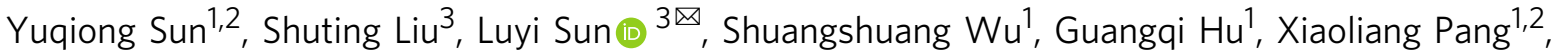
Andrew T. Smith ${ }^{3}$, Chaofan Hu ${ }^{1,2}$, Songshan Zeng ${ }^{3}$, Weixing Wang ${ }^{4}$, Yingliang Liu ${ }^{1,2 \times}$ \& Mingtao Zheng (1) ${ }^{1,2,3 凶}$

Room temperature phosphorescence materials have inspired extensive attention owing to their great potential in optical applications. However, it is hard to achieve a room temperature phosphorescence material with simultaneous long lifetime and high phosphorescence quantum efficiency. Herein, multi-confined carbon dots were designed and fabricated, enabling room temperature phosphorescence material with simultaneous ultralong lifetime, high phosphorescence quantum efficiency, and excellent stability. The multi-confinement by a highly rigid network, stable covalent bonding, and 3D spatial restriction efficiently rigidified the triplet excited states of carbon dots from non-radiative deactivation. The as-designed multi-confined carbon dots exhibit ultralong lifetime of $5.72 \mathrm{~s}$, phosphorescence quantum efficiency of $26.36 \%$, and exceptional stability against strong oxidants, acids and bases, as well as polar solvents. This work provides design principles and a universal strategy to construct metal-free room temperature phosphorescence materials with ultralong lifetime, high phosphorescence quantum efficiency, and high stability for promising applications, especially under harsh conditions.

\footnotetext{
${ }^{1}$ Key Laboratory for Biobased Materials and Energy of Ministry of Education/Guangdong Provincial Engineering Technology Research Center for Optical Agriculture, College of Materials and Energy, South China Agricultural University, Guangzhou 510642, China. ${ }^{2}$ Maoming Branch, Guangdong Laboratory for Lingnan Modern Agriculture, Maoming 525000, China. ${ }^{3}$ Polymer Program, Institute of Materials Science and Department of Chemical \& Biomolecular Engineering, University of Connecticut, Storrs, CT 06269, USA. ${ }^{4}$ Ministry of Education Key Laboratory of Enhanced Heat Transfer \& Energy Conservation,

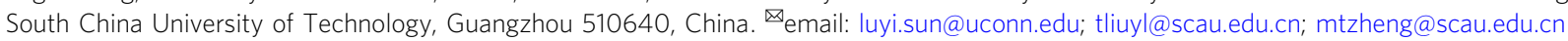


oom temperature phosphorescence (RTP) materials, as a typical class of long-lived emission materials, have evoked considerable attention during the past decade thanks to their promising applications in displays, chemical sensors, bioimaging, and anti-counterfeiting ${ }^{1}$. The present long-lived luminescent materials are mainly organic compounds through molecular design and inorganic complexes which are typically designed based on transition metals and rare-earth ions. Attractive RTP organics, which may enable conventional phosphorescence through the formation of specific structure (i.e., $\mathrm{H}$ aggregation, host-guest composition and polymer matrix $)^{2-5}$ to stabilize the triplet excitations or introducing special moieties (i.e., heavy halogen and aromatic aldehyde ${ }^{6,7}$ to enhance the intersystem crossing (Supplementary Fig. 1a). Nevertheless, the current organic RTP materials present short afterglow lifetime of several milliseconds, low stability, extreme sensitivity to oxygen/ vapor, and toxicity that cannot be neglected, which significantly limit their potential applications ${ }^{8-10}$. In contrast, traditional inorganic compounds exhibit superior long lifetime due to the unique exciton transition process, of which the emission is caused by slow release of trapped charge (traps formed by structure defects or complex impurities) by the thermal disturbance of ambient temperature (Supplementary Fig. 1b) ${ }^{11,12}$. However, given the scarcity of metal resources, complicated fabrication processes, and extreme instability in humid environments, none of these materials are ideal for permanent considerations. Especially, it is very difficult to simultaneously obtain an efficient RTP material with long afterglow lifetime and high quantum efficiency ${ }^{10,13}$. More recently, great efforts have been devoted to increasing quantum efficiency and prolonging lifetime of RTP materials by molecular design ${ }^{8,10,13-15}$. For instance, An's group made significant achievements to simultaneously enhance efficiency and lifetime of organic phosphorescence by molecular selfassembly ${ }^{8,10}$. Tang's group made remarkable progress on developing persistent and efficient pure organic RTP materials by intrinsic molecular-structure engineering or intramolecular triplet-triplet energy transfer ${ }^{13-15}$. However, current RTP materials still suffer from the short afterglow lifetime, low phosphorescence quantum efficiency (PQE), and poor stability. Therefore, exploring new generation of RTP materials with simultaneous long lifetime, high efficiency, and excellent stability through facile, green, and cost-effective methodology is highly desirable but remains a formidable challenge.

In the past decade, photoluminescence (PL) carbon dots (CDs) have triggered tremendous attention because of their facile preparation, high photo-stability, and low-toxicity ${ }^{16}$. Generally, CDs do not exhibit phosphorescence due to their unstable triplet excited states ${ }^{17,18}$. Great effort has been devoted to stabilizing triplet excited states of CDs through constructing hybrid systems of CDs in organic or inorganic matrices in order to achieve $\mathrm{RTP}^{17-21}$. Auxiliary matrices, including inorganic complexes (e.g., $\mathrm{KAl}\left(\mathrm{SO}_{4}\right)_{2} \cdot x \mathrm{H}_{2} \mathrm{O}, \mathrm{SiO}_{2}$, zeolites, layered double hydroxides (LDHs), etc.) ${ }^{17-19,21-25}$ and polymers/organic compounds (e.g., poly(vinyl alcohol), polyurethane, cyanuric acid, urea, etc.) ${ }^{26-29}$, were exploited to fabricate a matrix-assisted isolation structure. These substrates enable CDs to generate typical RTP emission by suppressing the intermolecular vibration (left in Fig. 1a) ${ }^{30,31}$. Unfortunately, current CD-based RTP materials generally face common troubles, including but not limited to, relatively short lifetime, low quantum efficiency, and poor stability, not to mention possess long lifetime and high efficiency simultaneously. Currently, there are three strategies to rigidify the triplets of CDs: (1) hydrogen bonds ${ }^{20}$. Early on researchers incorporated CDs into various matrices such as PVA to achieve RTP, in which hydrogen bonds formed between the CDs and the PVA matrix can protect the triplet excited states of CDs from rotational or vibrational loss. However, the strength of hydrogen bonds is relatively weak, thereby their effect on stabilizing triplet states is limited. (2) Covalent bonds ${ }^{20}$. Compared to weak hydrogen bonds, covalent bonds can be employed as a much more effective alternative to fix and rigidify triplet emission species. Therefore, researchers tried to incorporate CDs into colloidal silica through covalent bonds, and some significant features such as long afterglow and aqueous-related afterglow were observed ${ }^{17,22}$, since the emissive moieties could be fixed on certain substances by covalent bonds. However, in previous reports, the carbon and silicon used are usually derived from separate sources, and the recombination of the CDs with the silica matrices are generally carried out at low temperatures, which makes it difficult to form very stable C-Si bonds. (3) Structural confinements ${ }^{18,19,24}$. With further research progress, it was found that the incorporation of CDs into zeolites or two-dimensional LDHs can generate a structural confinement effect on CDs. However, CDs can only be embedded in the interrupted nano-space or attach to the external surface of zeolite host due to the larger size of CDs $(\approx 2-6 \mathrm{~nm})$ compared to the diameter of micropores (less than $2 \mathrm{~nm})^{19}$. Therefore, zeolites can only exert a relatively weak surface confinement to the embedded CDs, which is similar to the confinement effect by two-dimensional interlayer of LDHs, resulting in relatively short lifetime and low quantum efficiency. Generally, the phosphorescence lifetime of the reported CD-based RTP materials are mostly less than $2 \mathrm{~s}$, accompanied with a PQE less than $20 \%{ }^{32}$. In brief, the early work has shown that simply relying on one single confinement effect is not enough to achieve the desired RTP performance; breakthroughs on the phosphorescence lifetime, quantum efficiency, and stability of the CD-based RTP materials have not yet been made. Thus, exploration of design principles and fabrication strategies to effectively rigidify the triplet excited states of CDs for achieving a long-lived and efficient metal-free RTP material is highly desired but greatly challenging.

To achieve efficient RTP of CDs, some key features and principles must be satisfied in the fabrication strategy. Firstly, it is essential to construct a highly rigid network that can embed and isolate CDs from external quenching factors (such as oxygen, humidity, etc.). Secondly, it is vital to form stable covalent bonds between the matrix network and the embedded CDs to minimize the non-radiative decay of long-lived triplets. Thirdly, it is crucial to tightly fix the CDs in three-dimensional (3D) nano-space to produce an efficient spatial restriction, so as to effectively suppress the intramolecular vibration and stabilize the triplet excited states of CDs.

In this work, a strategy is designed and exploited to fabricate metal-free multi-confined $\mathrm{CDs}$ within $\left.\mathrm{SiO}_{2}(\mathrm{CDs@SiO})_{2}\right)$ with ultralong RTP lifetime, high PQE, and exceptional stability simultaneously, by constructing an effective multi-confinement effect (MCE). Our results demonstrate that this unique multiconfinement exerted by tetrahedral interstice is more effective than conventional surface confinement of zeolites and $\mathrm{LDHs}^{18,24}$. It is also different from the ones covalently linked to a colloidal silica derived from the hydrolysis of tetraethyl orthosilicate ${ }^{17}$. The as-designed multi-confined RTP of CDs@SiO ${ }_{2}$ not only exhibits an ultralong RTP lifetime of $5.72 \mathrm{~s}$ (more than $40 \mathrm{~s}$ to the naked eye), but also achieves a very high PQE of $21.30 \%$. Moreover, the as-fabricated $\mathrm{CDs@SiO}$. phosphors exhibit high stability, which can resist the quenching from strong oxidants, strong acids, and bases, water, as well as polar solvents. More importantly, this method can be easily expanded to other silicon-rich biomasses and other regular chemicals (instead of restricting to biomass only) to ensure a reproducible production of high quality multiconfined RTP materials with a potential for future large-scale manufacturing. Therefore, this work provides a universal strategy 
a

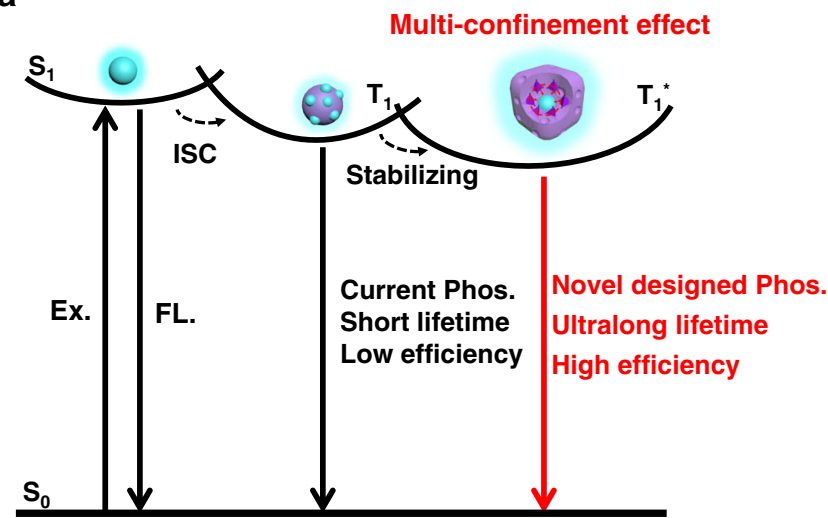

b

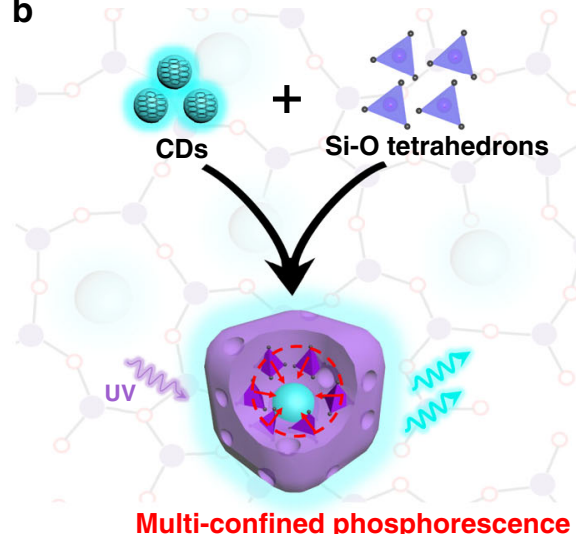

Fig. 1 Schematic of the proposed phosphorescence mechanism and design strategy of multi-confined CDs@SiO $\mathbf{2}_{\mathbf{2}}$ RTP material. a Proposed mechanism of CDs in conventional matrix and multi-confined structure. (SO ground state, S1 first excited singlet state, T1 first excited triplet state, ISC intersystem crossing, Ex. excitation, FL. fluorescence, Phos. phosphorescence). b Schematic of the design strategy of the multi-confined (rigid network, stable covalent bonds, and three-dimensional nano-space) phosphorescence.

to fabricate $\mathrm{CDs@SiO} 2$ phosphors and offers design principles and insights into metal-free RTP materials with ultralong lifetime, high quantum efficiency, and high stability simultaneously for various RTP-based applications.

\section{Results}

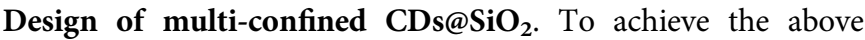
outlined principles, a strategy was developed to synthesize metalfree multi-confined RTP CDs@SiO $\mathrm{S}_{2}$ by constructing an effective MCE. First, rice husks (RHs) with a homogeneous distribution of carbon and silicon were employed as both the carbon and silicon source. By taking advantage of the intimate contact of carbon and silica in $\mathrm{RHs}^{33}$, we designed and achieved stable covalent interactions between the CDs and the $\mathrm{SiO}_{2}$ matrix. Second, a sol-gel process was developed to fabricate a vast $\mathrm{H}_{4} \mathrm{SiO}_{4}$ network linked by $\mathrm{Si}-\mathrm{O}-\mathrm{Si}$ chains. Note that the luminescent CDs were in situ generated and perfectly fixed within the polymerized network. Third, the subsequent high temperature calcination process not only results in a conversion of loose $\mathrm{H}_{4} \mathrm{SiO}_{4}$ to a rigid and compact silica network, but also forms stable covalent interaction between the CDs and the silica network, leading to the formation of multi-confined CDs. As depicted in Fig. 1a, b, CDs were in situ embedded into a vast $\mathrm{SiO}_{2}$ network composed of $\mathrm{Si}-\mathrm{O}$ tetrahedrons. Appropriate 3D nano-space surrounded by $\mathrm{Si}-\mathrm{O}$ tetrahedrons can tightly accommodate and embed CDs. The MCE consists of a highly rigid network, stable covalent bonding, and $3 \mathrm{D}$ spatial restriction that can effectively suppress the intramolecular vibration and thus successfully stabilizing the triplet excited states of the CDs.

Synthesis and characterization of $\mathrm{CDs@SiO}$. RHs are often treated as a biowaste, converting RHs biowaste into value-added products with broad applications remains a considerable challenge as well as a significant task ${ }^{33,34}$. In this work, we take advantage of the intimate contact of $\mathrm{C}$ and silica in RHs to design and fabricate $\mathrm{CDs@SiO}$ network with ultralong RTP lifetime, remarkable quantum efficiency, and extraordinary stability through an in situ method. As illustrated in Fig. 2, during the reflux pretreatment process, the $\mathrm{SiO}_{2}$ in $\mathrm{RHs}$ first reacted with $\mathrm{NaOH}$ to form $\mathrm{Na}_{2} \mathrm{SiO}_{3}$, as shown in Eq. 1. Simultaneously, some carbonaceous organics such as cellulose in RHs can be transferred into PL CDs at a temperature of $160^{\circ} \mathrm{C}^{35,36}$. The as-generated $\mathrm{Na}_{2} \mathrm{SiO}_{3}$ was then turned into mono-silicic acid $\left(\mathrm{H}_{4} \mathrm{SiO}_{4}\right)$ in an acidic environment (Eq. 2). The freshly generated $\mathrm{H}_{4} \mathrm{SiO}_{4}$ can be polymerized to form a vast network linked by $\mathrm{Si}-\mathrm{O}-\mathrm{Si}$ chains during the aging process ${ }^{25}$, which plays a crucial role in the whole preparation process, as shown in Eq. 3. Note that the luminescent CDs could be perfectly fixed inside the polymerized $\mathrm{H}_{4} \mathrm{SiO}_{4}$ network that protects the CDs from calcination at high temperature, forming spatially confined $\mathrm{CDs@SiO} 2$ phosphors with ultralong RTP and high stability. In order to optimize RTP luminescence, a series of experiments were conducted to fabricate $\mathrm{CDs@SiO}{ }_{2}-x$ ( $x$ refers to the calcination temperature) phosphors at various calcination temperatures. As shown in Supplementary Figs. 2 and 3 and Supplementary Table 1, the resultant $\mathrm{CDs@SiO} 2-600$ phosphors possess the ultralong phosphorescent lifetime and high quantum efficiency.

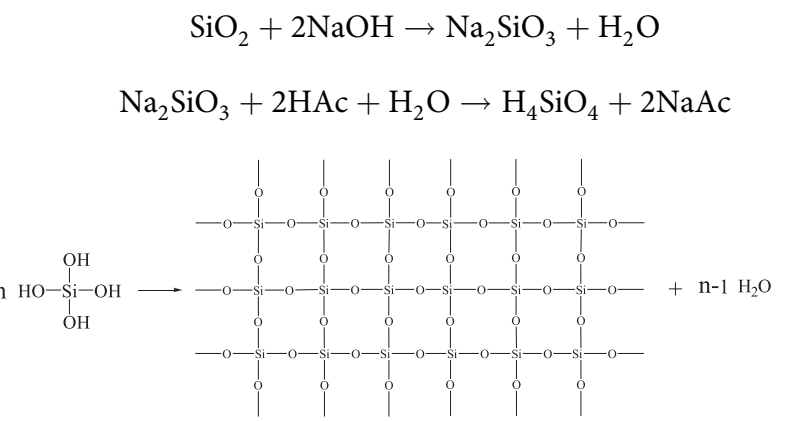

As shown in Fig. 3a, b and Supplementary Fig. 4, the transmission electron microscopy (TEM), high-resolution TEM (HRTEM) images, and X-ray diffraction (XRD) pattern of $\mathrm{CDs@SiO} \mathrm{S}_{2}-600$ reveal the existence of amorphous silica phase, that is the random network of Si-O tetrahedrons ${ }^{37}$, and the even distribution of $\mathrm{CDs}$ in the amorphous $\mathrm{SiO}_{2}$ network. The HRTEM image displayed in Fig. $3 \mathrm{~b}$ shows that the CDs embedded in $\mathrm{SiO}_{2}$ exhibit an average diameter of $3.0 \pm 0.5 \mathrm{~nm}$ (Fig. 3c) and a lattice fringe with a spacing of $0.21 \mathrm{~nm}$ (Fig. $3 \mathrm{~b}$ inset). Note that the pristine CDs derived from the mother liquor, through a dialysis, possess an average size of $4.8 \mathrm{~nm}$ (Supplementary Fig. 5a, b). The decrease of the particle size of the CDs might be due to the reduction of surface groups during the calcination process ${ }^{25}$.

To further deduce the chemical interactions between the CDs and the $\mathrm{SiO}_{2}$, Fourier transform-infrared spectroscopy (FTIR) and X-ray photoelectron spectroscopy (XPS) of CDs and CDs@SiO -600 were carried out. The FTIR spectrum (Fig. 3d) shows characteristic peaks at 1635 and $3438 \mathrm{~cm}^{-1}$ that can be 


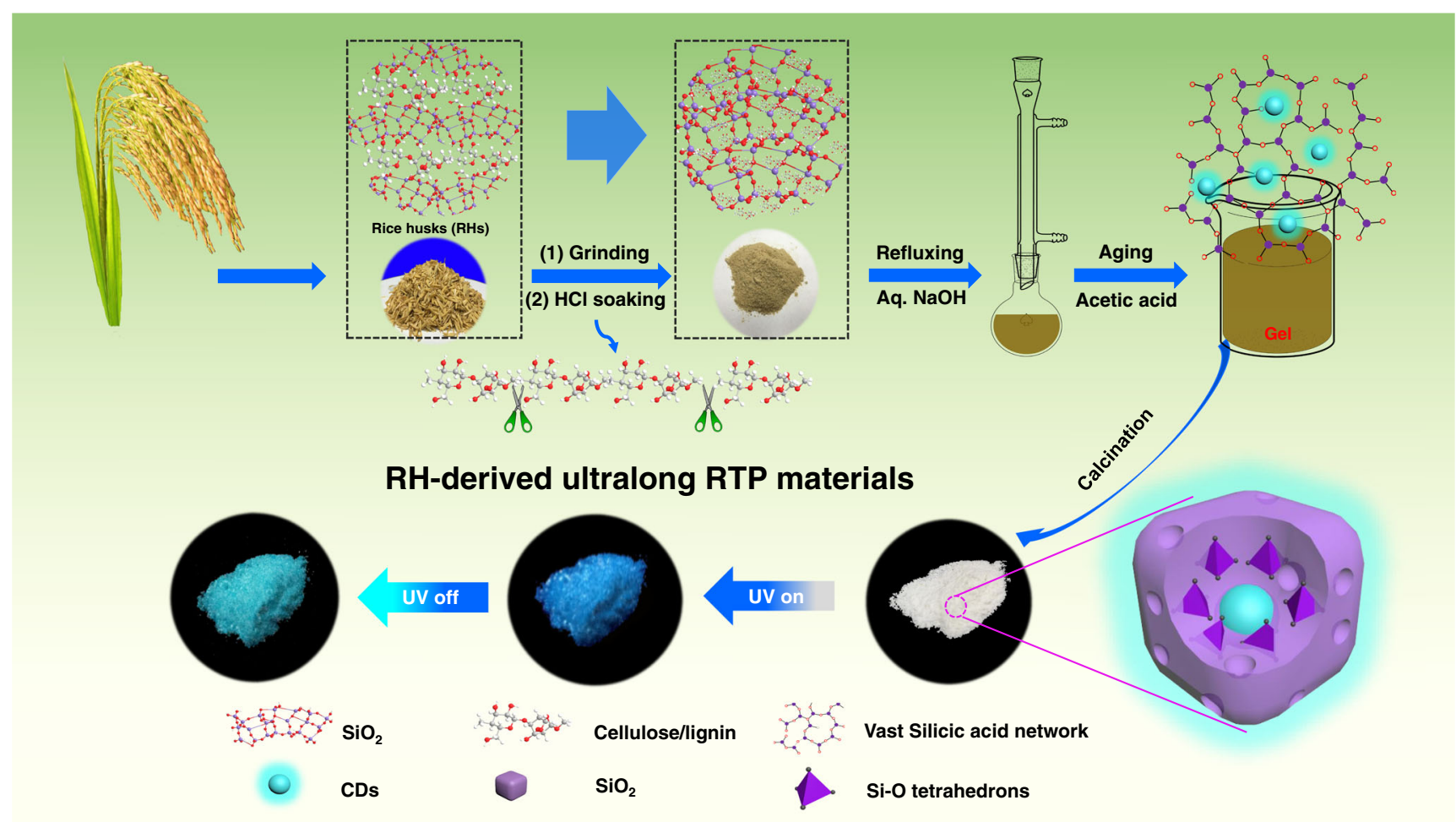

Fig. 2 Design of multi-confined CDs@SiO $\mathbf{2}_{2}$. Schematic of the overall process for fabrication of the metal-free CDs@SiO $\mathrm{CTT}_{2}$ materials with ultralong lifetime from rice husks ( $\mathrm{RHs}$ ).

attributed to $\mathrm{C}=\mathrm{O}$ and $\mathrm{O}-\mathrm{H}$ stretching vibrations, respectively. Importantly, the obvious peaks around $1106 \mathrm{~cm}^{-1}$ ( $\mathrm{Si}-\mathrm{O}-\mathrm{C}$ stretching vibrations) and $790 \mathrm{~cm}^{-1}$ (Si-C stretching vibrations) reveal the formation of covalent bonds between the CDs and the amorphous $\mathrm{SiO}_{2}$, suggesting that the $\mathrm{CDs}$ and the $\mathrm{Si}-\mathrm{O}$ network are connected by covalent bonds. The XPS results further confirm this hypothesis. The XPS survey scan in Fig. 3e shows that this nanocomposite mainly consists of $\mathrm{C}, \mathrm{O}$, and Si elements. The XPS C 1s spectra (Fig. 3f) indicated the presence of C-Si $(284.0 \mathrm{eV})$, $\mathrm{C}-\mathrm{C} / \mathrm{C}=\mathrm{C}(284.7 \mathrm{eV}), \mathrm{C}-\mathrm{O}(285.3 \mathrm{eV})$, and $\mathrm{C}=\mathrm{O}(290.0 \mathrm{eV})$ bonds ${ }^{22,28}$. The XPS O $1 \mathrm{~s}$ spectra (Fig. $3 \mathrm{~g}$ ) shows two peaks at 531.7 and $532.8 \mathrm{eV}$ that can be assigned to $\mathrm{C}=\mathrm{O}$ and $\mathrm{Si}-\mathrm{O}$ bonds, respectively ${ }^{25}$. The XPS Si $2 \mathrm{p}$ spectra (Fig. 3h) exhibits three binding energies at $102.6,103.2$, and $103.9 \mathrm{eV}$, indicating the presence of $\mathrm{Si}-\mathrm{C}, \mathrm{SiO}_{2}$, and $\mathrm{SiO}_{2}$ (gel), respectively ${ }^{22}$. The existence of $\mathrm{Si}-\mathrm{C}$ bonds was also verified in the samples calcined at other temperatures (Supplementary Fig. 6). Based on the above results, the structure and possible bonding between the CDs and the $\mathrm{SiO}_{2}$ network are illustrated in Fig. 3i. The initially synthesized $\mathrm{CDs}$ as luminescence centers with $\mathrm{C}=\mathrm{O}$ and $\mathrm{O}-\mathrm{H}$ functional groups (Supplementary Figs. 7 and 8) were embedded into the $\mathrm{SiO}_{2}$ matrix through both covalent and hydrogen bonds. Because of those bond interactions, the intermolecular motion of the CDs is prohibited and the long-lived phosphorescence occurs. Furthermore, this stable structure of $\mathrm{CDs@SiO} 2$ helps to suppress the non-radiative transition and makes it resistant to the quenching of strong oxidants, acids and bases, and polar solvents.

Optical properties of $\mathrm{CDs} @ \mathrm{SiO}_{2}$. The UV-vis absorption spec-

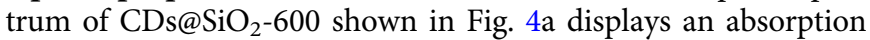
peak at $225 \mathrm{~nm}$ and a broad band from 240 to $370 \mathrm{~nm}$, which are attributed to the $\pi-\pi^{*}$ transition of $C=C$ and $n-\pi^{*}$ transition of $\mathrm{C}=\mathrm{O}$, respectively ${ }^{24}$. The PL excitation spectrum shows a broad band from 234 to $372 \mathrm{~nm}$ with the maximum excitation peaking at $260 \mathrm{~nm}$, highly overlapping with the absorption band of $\mathrm{C}=\mathrm{O}$ bonds, suggesting that the luminescence should come from the $\mathrm{C}$ $=\mathrm{O}$ bonds of CDs. The as-fabricated multi-confined RTP CDs@SiO -600 presents blue fluorescence emission and ultralong phosphorescence at the excitation wavelength of $260 \mathrm{~nm}$ (Fig. 4a). As shown in the phosphorescent two-dimensional excitationemission plot (Fig. 4b), the phosphorescence can be excited by wavelengths from 240 to $300 \mathrm{~nm}$, with the best RTP emission obtained under $260 \mathrm{~nm}$ excitation centered at $464 \mathrm{~nm}$. The best excitation wavelength of $\mathrm{CDs@SiO}-600$ is different from that of the pristine RH-derived CDs (Supplementary Fig. 9), indicating a blue shifting of excitation wavelength when CDs is embedded and restricted in silica matrix. Furthermore, the emission intensity rises with the color changing from blue to red, and the emission peaks show excitation wavelength independence. The color coordinates of the FL and phosphorescence correspond to $(0.178$, $0.266)$ and $(0.172,0.209)$, respectively, through the CIE (Commission International d'Éclairage) 1931 chromaticity coordinates (Fig. 4c).

The multi-confined RTP CDs@SiO ${ }_{2}-600$ exhibits intense bluegreenish RTP after switching off the $254 \mathrm{~nm}$ irradiation, which could last more than $40 \mathrm{~s}$ to the naked eye (Fig. $4 \mathrm{~d}$ and Supplementary Movie 1). To obtain insights into the multiconfined RTP properties of the CDs@SiO $2-600$, the phosphorescence decay spectra was further measured (Fig. 4e), which could be well fitted to a tri-exponential function. According to the following equation:

$$
\tau_{\mathrm{avg}}=\sum \alpha_{i} \tau_{i}^{2} / \sum \alpha_{i} \tau_{i}
$$

the calculated average lifetime is up to $5.72 \mathrm{~s}$. This is by far much higher than that of the previously reported CD-based RTP materials (Supplementary Table 2) ${ }^{18-32,38-43}$ and pure organic RTP materials ${ }^{1-15}$. The $\Delta E_{\mathrm{ST}}$ between the lowest triplet $\left(\mathrm{T}_{1}\right)$ and the lowest singlet $\left(\mathrm{S}_{1}\right)$ gap was calculated to be $0.155 \mathrm{eV}\left(\mathrm{S}_{1}=\right.$ $2.827 \mathrm{eV}$ and $\mathrm{T}_{1}=2.672 \mathrm{eV}$ ). Such a low value benefits effective ISC process to populate triplet excitations. 

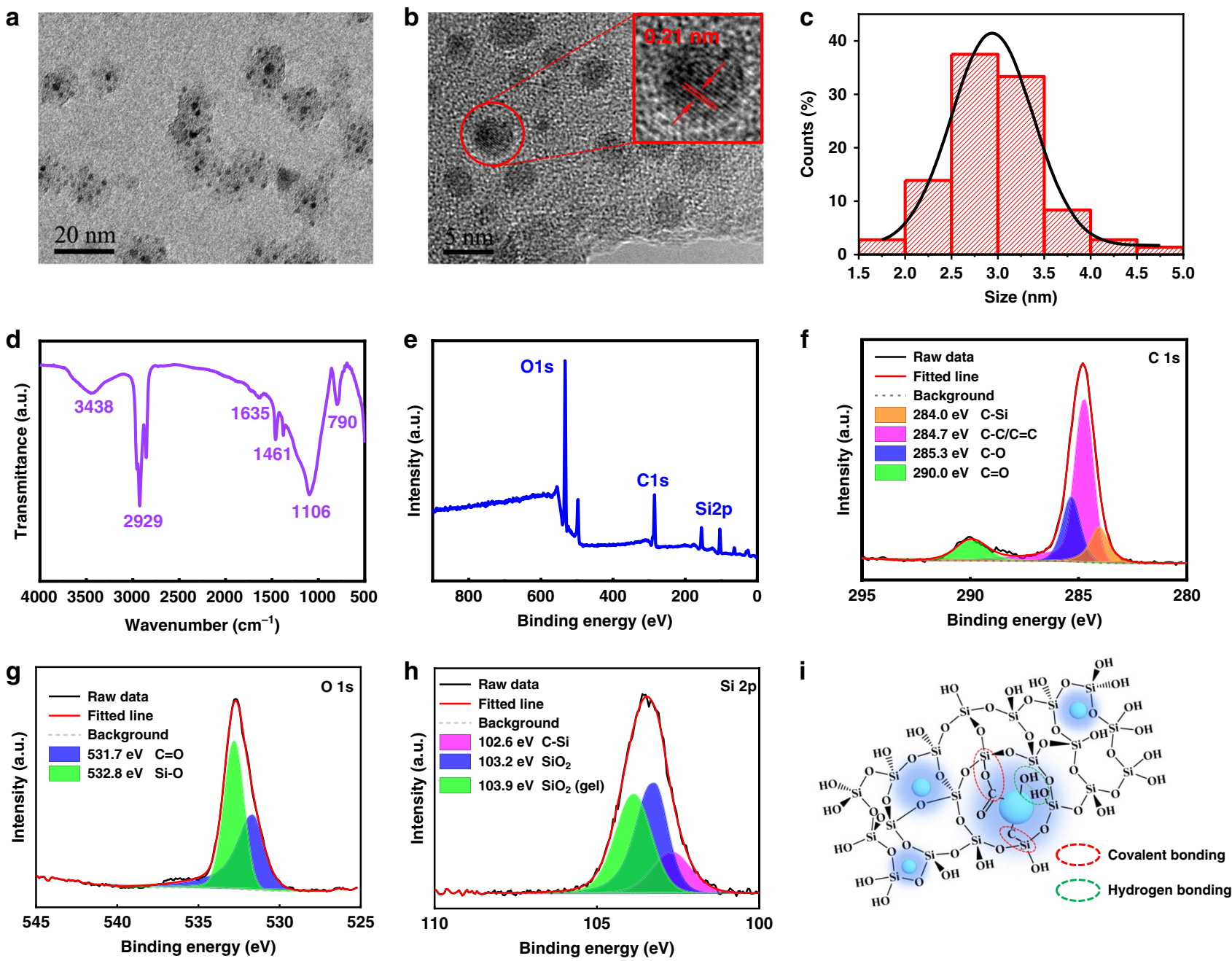

Fig. 3 Morphology, FTIR, and XPS characterization of the CDs@SiO $\mathbf{S}_{\mathbf{2}}$ a TEM and $\mathbf{b}$ HRTEM images of CDs@SiO ${ }_{2}-600$. c Size distribution histogram of the $\mathrm{CDs}$ in $\mathrm{SiO}_{2}$ matrix prepared at $600^{\circ} \mathrm{C}$. d FTIR spectrum and $\mathbf{e X P S}$ survey spectrum of $\mathrm{CDs} @ \mathrm{SiO}_{2}-600$. f-h High resolution XPS spectrum and fitting results of C 1s (f), O 1s (g), and Si 2p (h) in CDs@SiO $2-600$. i Schematic of the proposed structure of CDs@SiO $\mathrm{S}_{2}$.

The temperature-dependent phosphorescence emission and lifetime decay were further investigated (Fig. $4 \mathrm{f}$ and Supplementary Fig. 10). The RTP intensity and lifetime of $\mathrm{CDs@SiO} 2-600$ apparently decreased as the temperature increased due to the slow radiative decay of long-lived excited states and the non-radiation transition that is largely activated at elevated temperatures ${ }^{2,43,44}$, suggesting a phosphorescence characteristic rather than delayed fluorescence. Meanwhile, the full width at half-maximum (FWHM) of the emission spectra generally broadens due to the thermal expansion and electron-phonon interactions $s^{45}$. According to the temperature dependence phosphorescence emission, the FWHM was extended from 103 to $116 \mathrm{~nm}$ when the temperature increases from 77 to $350 \mathrm{~K}$. Such a slight change may be ascribed to the protection of MCE. It is worth mentioning that an ultralong phosphorescence lifetime (up to $3.43 \mathrm{~s}$ ) was achieved when the temperature was raised to $350 \mathrm{~K}$, which is still much higher than the previously reported state-of-the-art RTP materials (Supplementary Table 3 ). The understanding gained from the experimental results will help promote applications of the prepared $\mathrm{CDs@SiO} \mathrm{S}_{2}$ phosphors in high-temperature environment.

The emission from $\mathrm{CDs@SiO}$ 2 phosphors is hypothesized to originate from carbon luminescence centers immobilized in the $\mathrm{Si}-\mathrm{O}$ network, and the emission intensity also corresponds to the $\mathrm{CDs}$ content ${ }^{46}$. The carbonyl related groups on $\mathrm{CDs}$ can significantly improve the spin-orbit coupling to generate stable triplet excitons (unpaired electrons), thus resulting in phosphorescence emission. To verify our hypothesis, EPR spectra of the samples prepared at different calcination temperatures were recorded to characterize the stable triplet states. As shown in Fig. $4 \mathrm{~g}$, the intensity of the EPR signal at $240-260 \mathrm{G}$ decreases with an increasing annealing temperature, indicating that the amount of luminescence centers gradually decreases with the increase of annealing temperature. Because the CDs are confined in the interstices between $\mathrm{Si}-\mathrm{O}$ tetrahedrons, the spatial protection and restriction effect of the network protects the CDs from damage during the calcination process within an optimum temperature range. It is worth mentioning that the large numbers of carbon constituents in $\mathrm{Si}-\mathrm{O}$ network calcined at low temperatures (e.g., $400{ }^{\circ} \mathrm{C}$ ) are not beneficial to the luminescence. First, the high content of CDs might cause the high concentration annihilation characteristic. Moreover, we propose that the phosphorescence performance of $\mathrm{CDs} @ S i O_{2}$ was not only influenced by the content of CDs, but largely affected by the multi-confinement structure. Although $\mathrm{CDs}_{\mathrm{S}} \mathrm{SiO}_{2}-400$ had the largest numbers of luminescence centers, the weak spatial confinement caused by the loose $\mathrm{Si}-\mathrm{O}$ network renders them to exhibit relatively poor phosphorescence performance. Meanwhile, if the calcination temperature is too high $\left(700^{\circ} \mathrm{C}\right)$, less luminescence centers are left, which also results in weaker emission ${ }^{47}$. The TGA curve of the gel can further verify the 
a

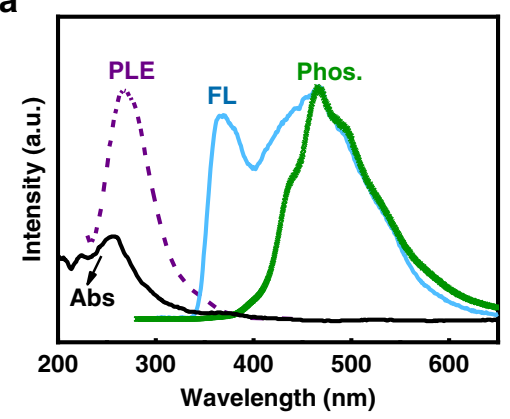

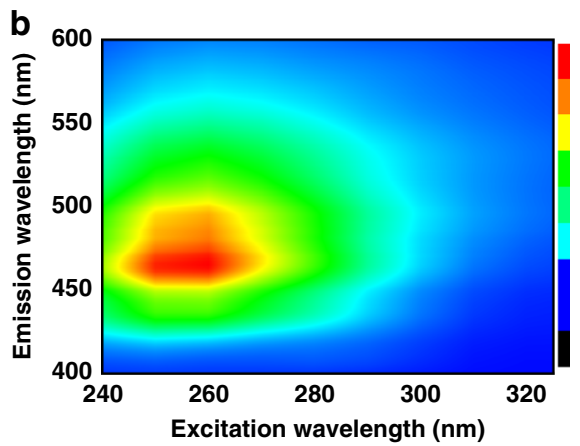
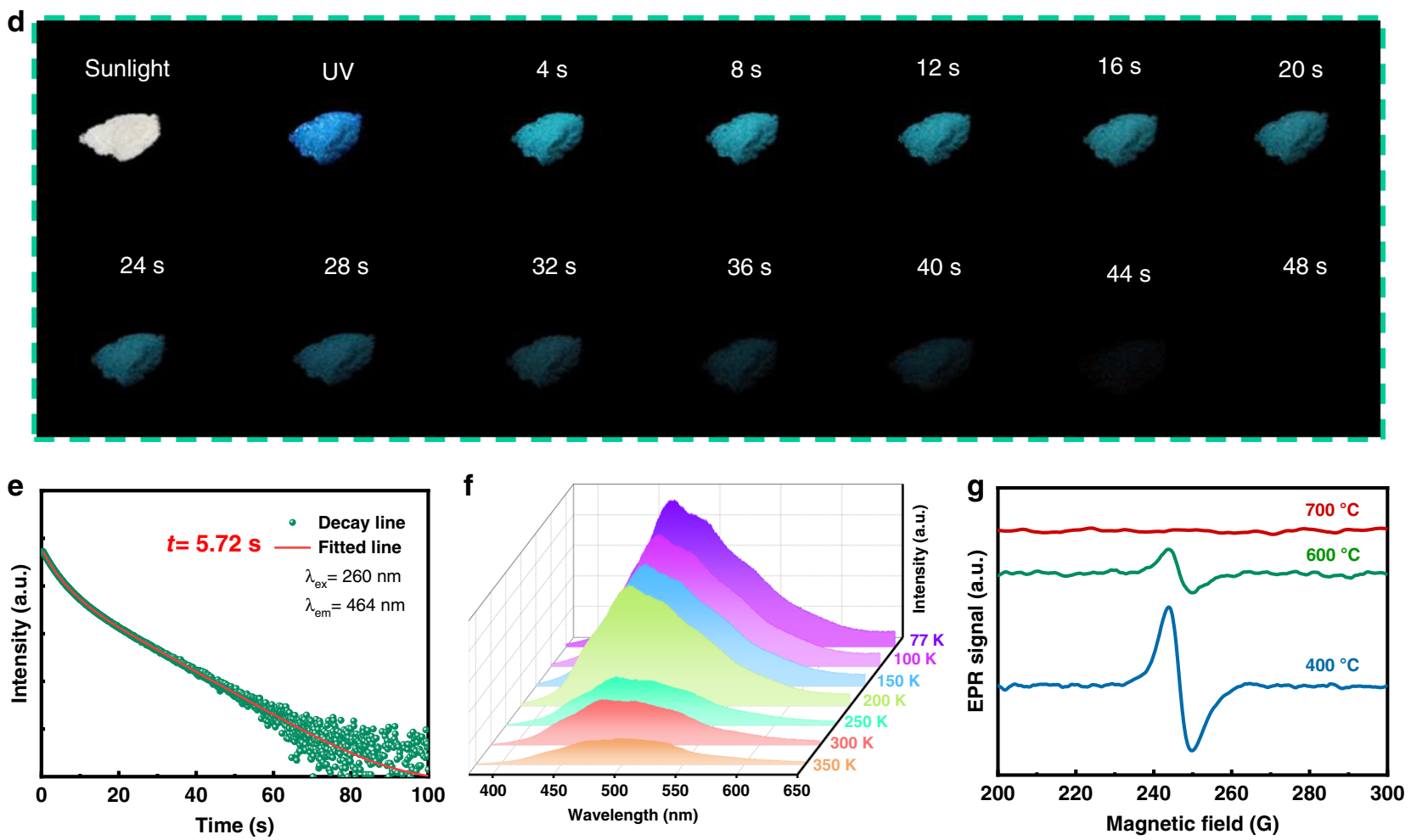

Fig. 4 Optical properties of CDs@SiO2. a Absorption, photoluminescence excitation, fluorescence emission, and phosphorescence emission spectra of $\mathrm{CDs@SiO}-600$. b Phosphorescent two-dimensional excitation-emission plot of $\mathrm{CDs}_{2} \mathrm{SiO}_{2}-600$. Emission intensity rises with the color changing from blue to green and to red. c CIE coordinates of the fluorescence emission and phosphorescence of $\mathrm{CDs}_{2} @ \mathrm{SiO}_{2}-600$. d Photographs of CDs@SiO -600 powders under sunlight, excited with $254 \mathrm{~nm}$ UV lamp, and after switching off UV. e Time-resolved phosphorescence decay and fitting curve (red line) of the

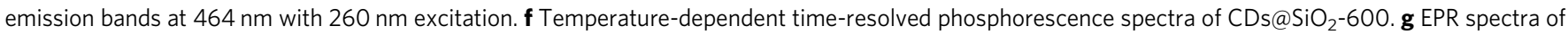
CDs@SiO $-400, \mathrm{CDs} @ \mathrm{SiO}_{2}-600$, and CDs@SiO -700 .

change of carbon contents (Supplementary Fig. 11). The weight of the gel reduced ca. $7.8 \%$ when the temperature was continuously increased from 400 to $600{ }^{\circ} \mathrm{C}$, and then further decreased by $1.1 \%$ up to $700^{\circ} \mathrm{C}$. Therefore, a suitable calcination temperature $\left(600^{\circ}\right.$ C) is important to obtain excellent ultralong-lived RTP materials.

According to the above results, we propose that such an ultralong RTP is simultaneously attributed to the MCE of the interstices between $\mathrm{Si}$ and $\mathrm{O}$ tetrahedrons, which can not only anchor CDs to provide unique stability, but also exert an effective spatial restriction to suppress the intramolecular vibration and rigidify the triplet excited state of the CDs, as illustrated in Supplementary Fig. 12. It is noteworthy that high temperatures over $700^{\circ} \mathrm{C}$ will lead to the collapse of these nano-space surrounded by $\mathrm{Si}-\mathrm{O}$ tetrahedrons, resulting in the weakening of the MCE. The sample calcined at $300^{\circ} \mathrm{C}$, exhibiting no phosphorescence, also further verifying the structural state of the CDs@SiO $\mathrm{S}_{2}$. But when recalcined at higher temperatures, the ultralong RTP was achieved again. This phenomenon suggests that the loose $\mathrm{Si}-\mathrm{O}$ network cannot confine CDs well, but with an increasing calcination temperature, the loose structure converts into a compact short-range ordered state, which is necessary to exert a spatial restriction to the embedded CDs, as illustrated in Supplementary Fig. 12. Another evidence is that the XRD pattern shows that the $\mathrm{CDs@SiO}$ changes from amorphous to crystalline as the calcination temperature increases, which further indicates the formation of short-range ordered transition state between the amorphous and crystalline (Supplementary Fig. 3). To further highlight the MCE in multi-confined RTP CDs@SiO ${ }_{2}$, a CDs/n$\mathrm{SiO}_{2}$ nanocomposite was prepared by mixing nano silica sol (n$\mathrm{SiO}_{2}$ ) with $\mathrm{RH}$-derived $\mathrm{CDs}$ directly and then subjected to the same calcination treatment. As shown in Supplementary Fig. 13, the $\mathrm{CDs} / \mathrm{n}-\mathrm{SiO}_{2}$ nanocomposite exhibits very weak phosphorescence that is barely captured because the CDs cannot be well embedded within the network structure of the pre-synthesized silica. These results suggest that the MCE of $\mathrm{Si}-\mathrm{O}$ network is crucial to obtain ultralong phosphorescence. 
Furthermore, the resultant $\mathrm{CDs} @ \mathrm{SiO}_{2}$ phosphors present very high absolute PQE of over $21 \%$ with ultralong lifetime (up to 5.72 s) simultaneously. The maximum absolute PQE of the resultant multi-confined RTP CDs@SiO 2 reached 26.36\%, which is much higher than most of the previously reported metal-free RTP phosphors of CDs in various matrices ${ }^{18-32,38-43}$ and organic RTP materials ${ }^{1-15,43,48-51}$ (Table S2). The high PQE may also be due to the special MCE. A highly rigid environment constructed by the $\mathrm{Si}-\mathrm{O}$ network plays a significant role in isolating CDs from external quenching factors. Meanwhile, the stable interaction between Si and O network and CDs benefits to minimize the nonradiative decay of long-lived triplets. It is worth mentioning that the PQE gradually decreases as the calcination temperature increases, which is probably owing to the influence of temperature on MCE and the reduction of CDs. When the calcination temperature reaches 500 and $600{ }^{\circ} \mathrm{C}$, the quantum efficiency can reach high values of $26.36 \%$ and $21.30 \%$, respectively. It can be observed from the TGA thermogram (Supplementary Fig. 11) that the content of CDs barely decreases between 500 and $600{ }^{\circ} \mathrm{C}$, and thus the main reason for the decrease of PQE should be the change of the spatial confinement structure. It is found that the PQE gradually decreases along with the further increase in temperature due to the reduction of CDs, and thus the quantum efficiency is only $15 \%$ at $650{ }^{\circ} \mathrm{C}$. When the temperature gradually increases to $700{ }^{\circ} \mathrm{C}$, the matrix of silica partially collapses and the content of CDs decreases, leading to the decrease of the PQE and phosphorescence emission intensity.

Stability of the resultant $\mathrm{CDs@SiO}$ phosphors. Thanks to the protection by $\mathrm{Si}-\mathrm{O}$ network, the $\mathrm{CDs@SiO} 2$ phosphors exhibit excellent anti-quenching properties and exceptional stability. As shown in Fig. 5a, the as-prepared $\mathrm{CDs@SiO} 2$ powders were first dispersed in strong oxidants, including $\mathrm{H}_{2} \mathrm{O}_{2}$ (30 wt\%), concentrated $\mathrm{HNO}_{3}$ (30 wt\%), and $\mathrm{H}_{2} \mathrm{SO}_{4}$ (98 wt\%), and then ultrasonicated for $30 \mathrm{~min}$. It is surprising that the PL is not quenched after being treated by strong oxidants, though the phosphorescence lifetime was slightly decreased, but a very long lifetime afterglow can still be observed with the naked eye (Fig. 5d, h). In order to further investigate the extraordinary stability of the $\mathrm{CDs@SiO}$. phosphors, they were also dispersed in different polar solvents and treated in a similar way. It was found that the phosphors also maintained superior stability in organic polar solvents (Fig. 5b, e, g). To further highlight the remarkable stability, the $\mathrm{CDs@SiO} 2$ phosphors were similarly treated in aqueous solutions at $\mathrm{pH}$ values varying from 1.0 to 14.0. As shown in Fig. 5c and Supplementary Fig. 14, the phosphorescence emission intensity only changed slightly in the $\mathrm{pH}$ range of 1.0 to 12.0, which indicates that the $\mathrm{CDs@SiO} 2$ phosphors can resist strong acids and strong bases. It is known that a concentrated base can corrode silica and thereafter potentially deteriorate the phosphorescence intensity. However, even after being treated by a strong base with a pH of 14.0, the afterglow of the $\mathrm{CDs@SiO}$ phosphors still remains basically unchanged while only the phosphorescence intensity decreases (Fig. 5f-h), further highlighting the protective effect of the Si-O network on the triplet state of CDs. These results indicate that the fabricated $\mathrm{CDs} @ \mathrm{SiO}_{2}$ phosphors manifest an exceptional stability, thanks to the protection by the $\mathrm{SiO}_{2}$ network to prevent the quenching of the CDs, making the $\mathrm{CDs@SiO} 2$ phosphors suitable for various applications especially in harsh environments.

Universality of the design principles. The above experimental results demonstrate that this work provides a facile and costeffective method to fabricate metal-free $\mathrm{CDs@SiO} 2$ phosphors with ultralong lifetime, high phosphorescence efficiency, and exceptional stability from $\mathrm{RH}$ biomass. More importantly, this method can be easily expanded to other silicon-rich biomasses, such as wheat husks (WHs) and Indocalamus leaves (ILs). As shown in Supplementary Fig. 15, the as-prepared WH-derived and IL-derived phosphors also exhibit ultralong RTP lifetime of $5.31 \mathrm{~s}$ and 3.95 , respectively. We propose that the cause of this

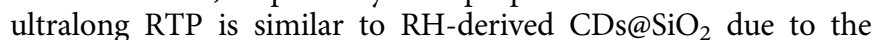
analogous structure and chemical component between RHs and WHs, which is attributed to the MCE of the interstices between $\mathrm{Si}$ and $\mathrm{O}$ tetrahedrons that can exert a spatial restriction to effectively rigidify the triplet excitons of CDs. These results demonstrate that the present work provides a universal green and lowcost approach to fabricate efficient metal-free RTP materials from natural biomass.

Since the successful achievement of efficient RTP from various silica-based biowaste, we propose that it is possible to achieve efficient RTP by incorporating CDs into the Si-O network using general chemical reagents as starting materials. To further verify our hypothesis and universality of this strategy for fabrication of efficient multi-confined RTP materials, $\mathrm{CD} 1 / \mathrm{SiO}_{2}, \mathrm{CD} 2 / \mathrm{SiO}_{2}$, and $\mathrm{CD} 3 / \mathrm{SiO}_{2}$ composites were fabricated by incorporating the three previously reported CDs into $\mathrm{SiO}_{2}$ by using $\mathrm{Na}_{2} \mathrm{SiO}_{3}$ and glacial acetic acid as precursors via a sol-gel process similar to CDs@SiO ${ }_{2}$ (please see the Supplementary methods for the detailed experimental procedures). As shown in Supplementary Fig. 16, $\mathrm{CD} 1 / \mathrm{SiO}_{2}, \mathrm{CD} 2 / \mathrm{SiO}_{2}$, and $\mathrm{CD} 3 / \mathrm{SiO}_{2}$ composites all exhibit blue-greenish RTP with lifetime of 4.92, 5.25, and $2.79 \mathrm{~s}$, respectively, indicating the feasibility of this MCE to rigidify and suppress the intramolecular vibration of CDs. The success to prepare $\mathrm{CD} / \mathrm{SiO}_{2}$ with efficient phosphorescence and exceptional stability using regular chemicals (instead of RHs biomass only) ensures the high quality, universality, and reproducibility for potential future large-scale production of multi-confined RTP materials.

Luminous mechanism of $\mathrm{CDs@SiO}$. Based on the above results, a possible mechanism for realizing such ultralong lifetime and ultrahigh quantum efficiency multi-confined RTP $\mathrm{CDs@SiO}_{2}$ with high stability is clearly proved. The CDs first in situ fabricated and incorporated into the vast silicic acid network, and then the calcination process converts such framework into a compact $3 \mathrm{D}$ network interconnected by $\mathrm{Si}-\mathrm{O}$ tetrahedrons. The rigid network can isolate CDs from external quenchers, the stable covalent and hydrogen bonds between the rigid structure and CDs can stabilize the triplet excited states of CDs. More importantly, the sufficient 3D nano-space surrounded by $\mathrm{Si}-\mathrm{O}$ tetrahedrons exerts spatial restriction that can suppress the intramolecular vibration and thus effectively stabilizing the triplet excited states of the CDs. In addition, it also endows $\mathrm{CDs@SiO} 2$ with extraordinary stability against oxidants, strong acid and base, and others. This MCE is responsible for this ultralong lifetime and ultrahigh PQE, very different from the previously reported single hydrogen bonds, covalent bonds, or twodimensional confined interface ${ }^{18,22,24,28}$.

Time-resolved anti-counterfeiting applications. Given the ultralong phosphorescence emission up to $40 \mathrm{~s}$ to the naked eye and high stability, the $\mathrm{CDs@SiO}$. phosphors can be employed as an ideal material for use in time-resolved anti-counterfeiting, because such a long time of phosphorescence is very rare and hard to duplicate. As shown in Fig. 6a, we simply designed an

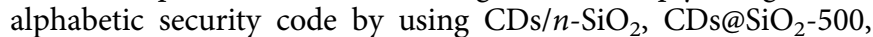
CDs@SiO -600 , and $\mathrm{CDs@SiO} 2-550$. Under the UV irradiation, the white pattern of "uconn" in daylight exhibit blue FL. Once stopping the excitation, because of the different phosphorescence 
a
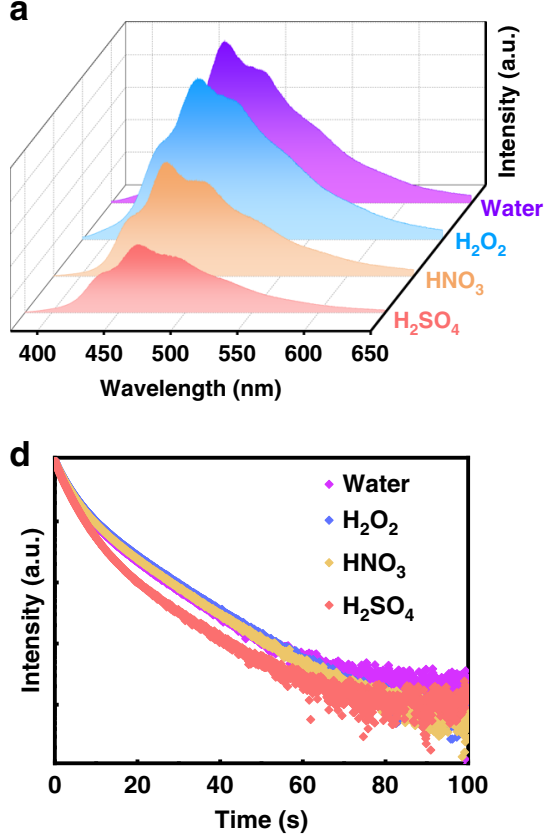

b

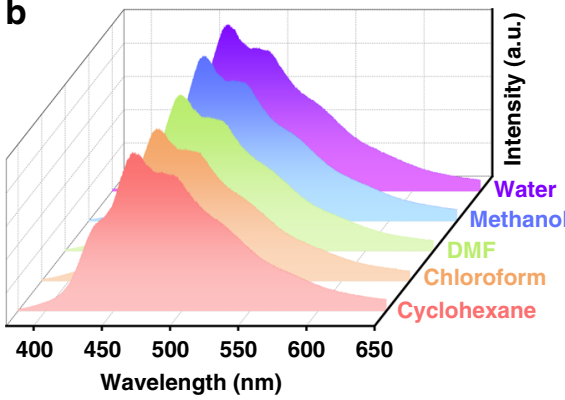

e

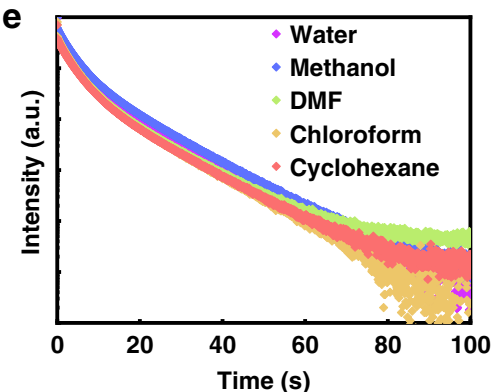

C
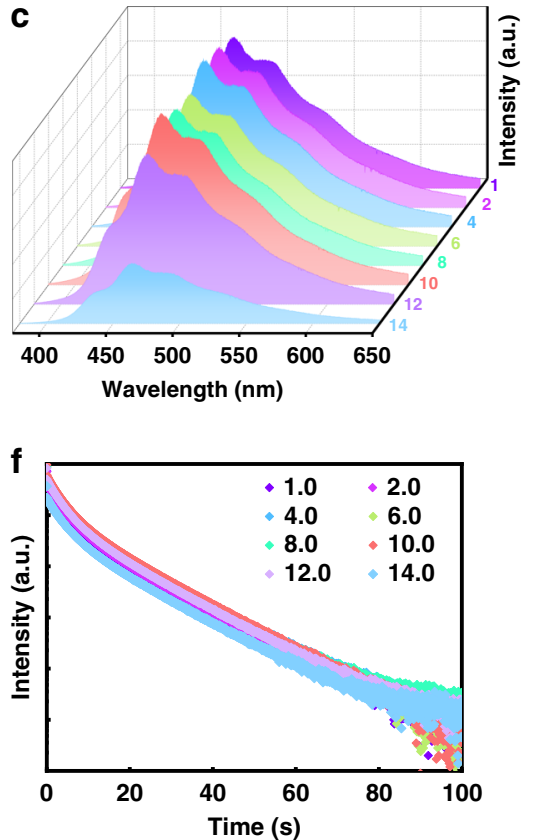

$9 8 \longdiv { 1 }$

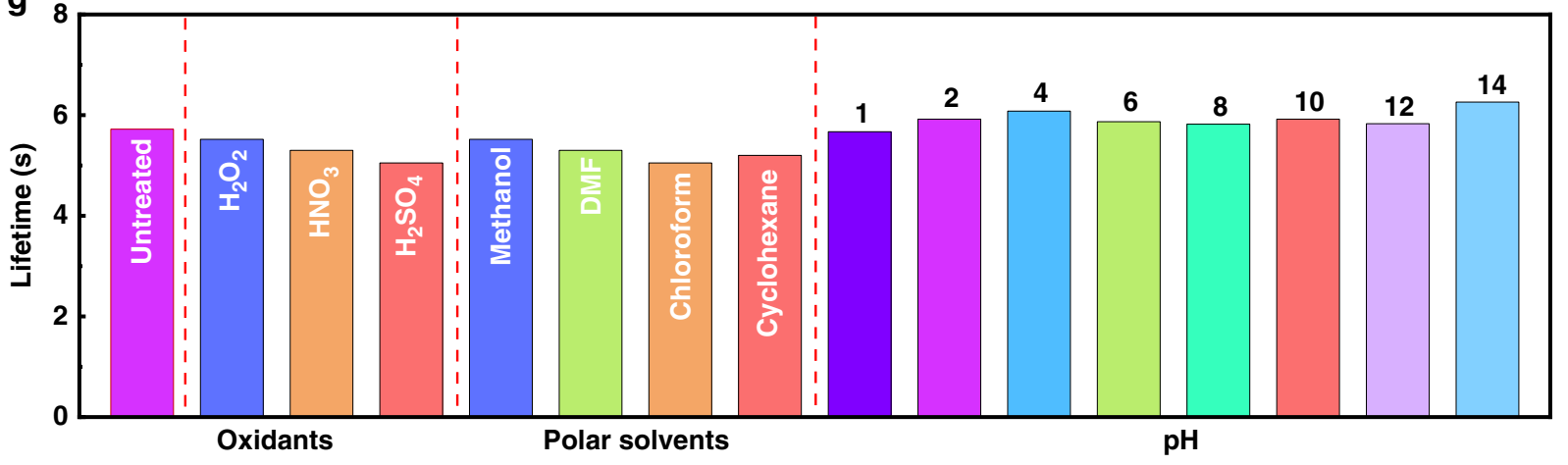

h
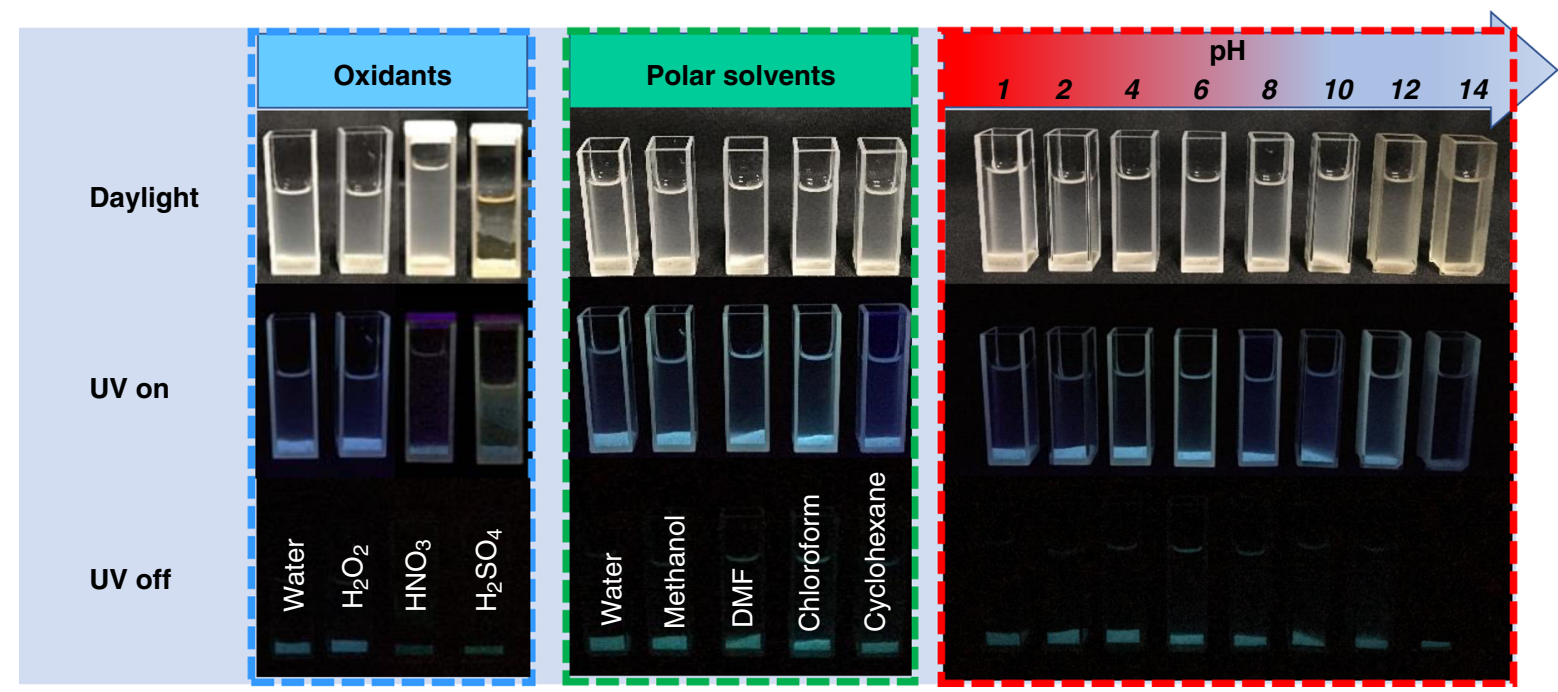

Fig. 5 Stability of the resultant CDs@SiO 2 against strong oxidants, strong acids and bases, water, and polar solvents. a, $\mathbf{d}$ Phosphorescence spectra and

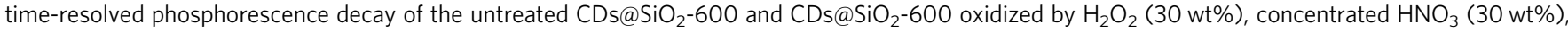

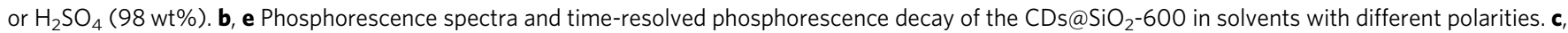
f Phosphorescence spectra and time-resolved phosphorescence decay of the $\mathrm{CDs} @ \mathrm{SiO}_{2}-600$ under pH values from 1.0 to 14.0. g Phosphorescent lifetime histogram of $\mathrm{CDs} @ \mathrm{SiO}_{2}-600$ in various oxidants, polar solvents, and pH values from 1.0 to 14.0. h Corresponding photographs of $\mathrm{CDs}_{0} @ \mathrm{SiO}_{2}-600$ in various oxidants, polar solvents, and water at different $\mathrm{pH}$ under sunlight, UV on and off. 


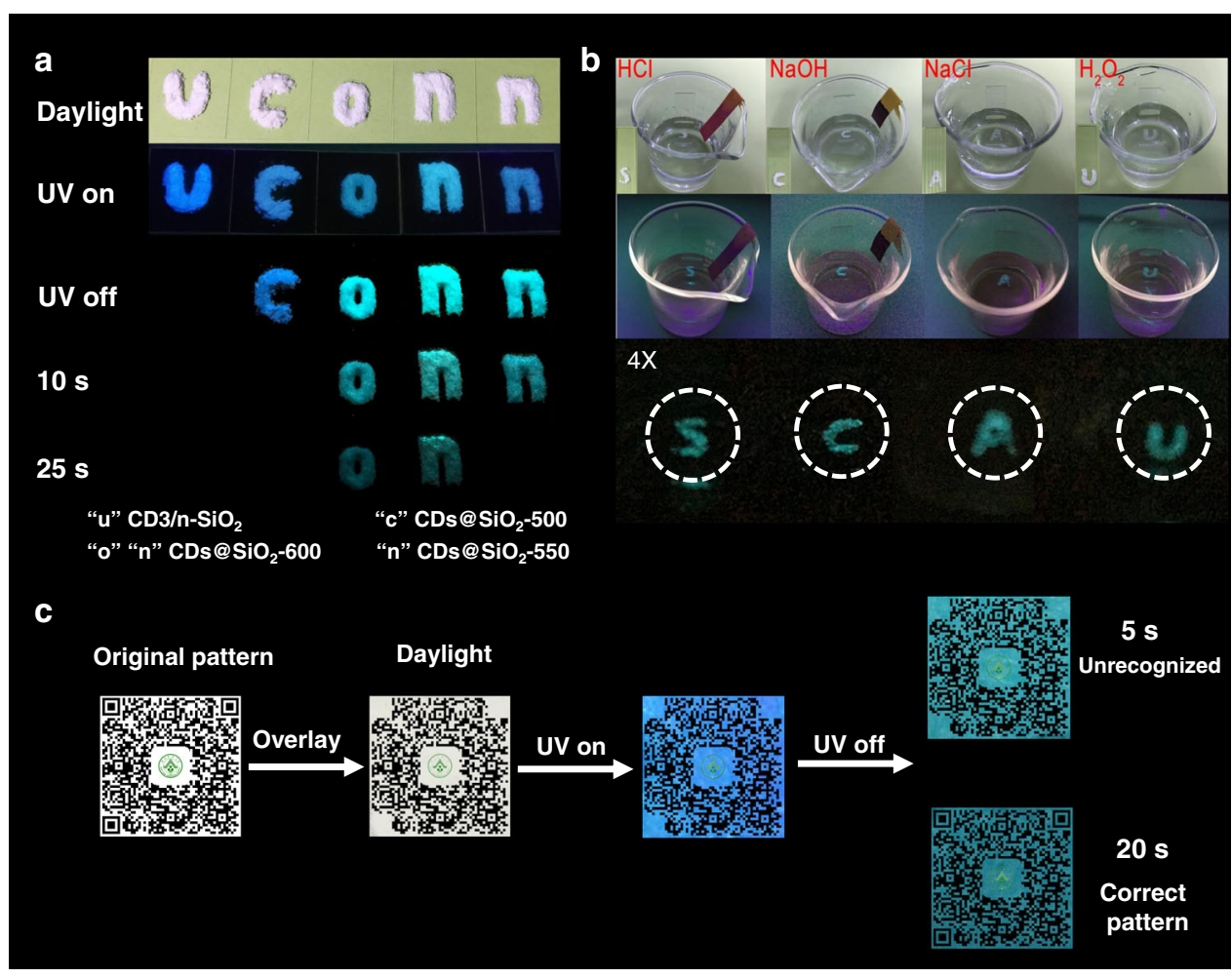

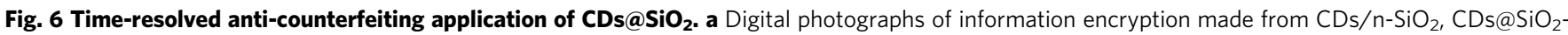
500,CDs@SiO $2-600$, and CDs@SiO $2-550$. b Digital photographs of phosphorescence from $\mathrm{CDs}_{2} @ \mathrm{SiO}_{2}-600$ in different environmental liquids. c Digital photographs of information encryption made from CDs@SiO $2-600$ and $\mathrm{CDs}_{2} \mathrm{SiO}_{2}-550$. The white area was overlaid with $\mathrm{CDs}_{2} @ \mathrm{SiO}_{2}-600$, and the black part of the corner is covered with CDs@SiO $2-550$.

emission time from $\mathrm{CDs@SiO} 2$ and free phosphorescence observed from $\mathrm{CDs} / n-\mathrm{SiO}_{2}$, only "conn" was shown. Furthermore, as time goes on to 10 and $25 \mathrm{~s}$, the " $\mathrm{c}$ " and the last " $\mathrm{n}$ " gradually disappeared, respectively. The encrypted pattern "on" was finally left behind after a layer of time screening (more than $25 \mathrm{~s})$. These results indicate the great promise of the as-fabricated $\mathrm{CDs@SiO} 2$ phosphors with ultralong lifetime for potential applications in the field of time-resolved encryption.

Figure $6 \mathrm{~b}$ shows the patterns of "S" " $C$ " " $A$ " " $U$ " immersed in different environmental liquids including $\mathrm{HCl}(2 \mathrm{M}), \mathrm{NaOH}(2$ M), $\mathrm{NaCl}(2 \mathrm{M})$, and $\mathrm{H}_{2} \mathrm{O}_{2}$ (30\%). Lasting blue-greenish phosphorescence after stopping the excitation can be observed,

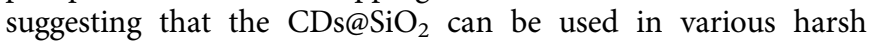

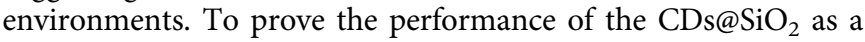
unique anti-counterfeiting tool, a QR (quick response) code with implicit information was also fabricated. As illustrated in Fig. 6c,

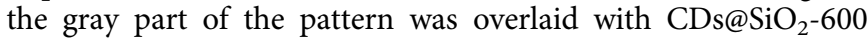
powders. To more distinctly compare the phosphorescence, CDs@SiO -550 was also employed to cover three black corners of the code. Under the daylight or a $254 \mathrm{~nm}$ UV lamp, the QR code cannot be identified to provide any information because the identification division was partially covered. Furthermore, it is interesting to observe that the phosphorescence from covering the corner with $\mathrm{CDs@SiO}-550$ has already quenched while the gray

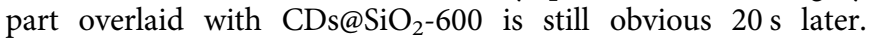
Therefore, when the UV lamp was switched off, an unrecognizable incomplete pattern was first obtained. Then the black part of the corner gradually appears along the time, and eventually a correct blue-greenish pattern is visible after $20 \mathrm{~s}$, which could be recognized by a scanner. The as-prepared special multi-confined RTP $\mathrm{CDs@SiO} 2$ phosphors with ultralong lifetime, ultrahigh quantum efficiency, and high stability are also expected to find applications in biomedical, optoelectronic, etc.

\section{Discussion}

In summary, a room-temperature phosphorescence material of multi-confined CDs simultaneously with ultralong lifetime, high quantum efficiency, and excellent stability is designed and fabricated by a universal strategy. An ultralong RTP emission with lifetime up to $5.72 \mathrm{~s}$ (more than $40 \mathrm{~s}$ to the naked eye) and high PQE of $21.30 \%$ can be simultaneously achieved through MCE originated from $\mathrm{Si}$ to $\mathrm{O}$ network. The maximum absolute PQE of the resultant phosphors can reach $26.36 \%$. Furthermore, the multiconfined CDs@SiO ${ }_{2}$ phosphors show exceptional stability in strong oxidant agents (i.e., $\mathrm{H}_{2} \mathrm{O}_{2}$, concentrated $\mathrm{HNO}_{3}$, and $\mathrm{H}_{2} \mathrm{SO}_{4}$ ), organic solvents with different polarities, strong acids, and strong bases. The experiment results suggest that the MCE of highly rigid network, stable covalent/hydrogen bonds, and sufficient 3D nanospace is responsible for this ultralong lifetime, high quantum efficiency, and extraordinary stability of the as-designed CD-based multi-confined RTP material. We later verified that multi-confined RTP materials can be synthesized using regular chemicals via this universal strategy. The findings offer design principles and insights to fabricate metal-free RTP materials for promising applications in biomedicine, optical anti-counterfeiting, and optoelectronics, especially for applications under extreme environments.

\section{Methods}

Materials. The RHs were collected from a mill in Guangzhou, China. Glacial acetic acid $(\mathrm{HAc})$ and hydrochloric acid $(\mathrm{HCl})$ were purchased from Guangzhou Chemical Reagent Co., Ltd., and sodium hydroxide $(\mathrm{NaOH})$ was purchased from Guangdong Guanghua Sci-Tech Co., Ltd. Deionized water used in this project was supplied by a Water Purifier Nano pure water system (Master-E, Hitech-Science tool, Shanghai, China). All reagents are of analytical grade and were used without further purification.

Synthesis of CDs@SiO 2 RTP materials. In a typical process, $10 \mathrm{~g}$ of dried RHs were first ground into fine powders $(200 \mathrm{mesh})$, soaked with a $\mathrm{HCl}$ solution $(2.0 \mathrm{M})$ 
for $2 \mathrm{~h}$ under magnetic stirring, and then washed to neutral with deionized water. Subsequently, the $\mathrm{HCl}$ pretreated $\mathrm{RHs}$ were refluxed in $100 \mathrm{~mL}$ of $\mathrm{NaOH}$ solution $(0.8 \mathrm{M})$ in a round bottom flask with magnetic stirring at $160^{\circ} \mathrm{C}$ for $6 \mathrm{~h}$. Then, the mixture was filtered to obtain the $\mathrm{CD}$-containing mother liquor. HAc was added drop-wise into the aforementioned mother liquor under magnetic stirring, until the $\mathrm{pH}$ reached 5-6 to ensure proper gelation. The as-resulted solution was then aged for $6 \mathrm{~h}$ to form a chocolate-brown solid gel, which was then washed with deionized water and ethanol to remove surface-attached CDs and inorganic salts. After that, the gel was dried at $60^{\circ} \mathrm{C}$ in a vacuum oven for $10 \mathrm{~h}$ and ground into fine powders for further use.

The as-prepared gel powders were calcined in air in a furnace with a ramp rate of $5{ }^{\circ} \mathrm{C} / \mathrm{min}$ to obtain $\mathrm{CDs} @ \mathrm{SiO}_{2}$ composites. During the calcination process, the optimum processing condition was determined by calcining various temperatures in the range of $400-700^{\circ} \mathrm{C}$ for $90 \mathrm{~min}$. Hereinto, the samples were denoted as $\mathrm{CDs@SiO} 2-x$, where $x$ refers to the calcination temperature.

Characterization. UV-vis absorption spectra of the samples were recorded on a Shimadzu UV-2550 ultraviolet-visible spectrophotometer. XRD (Rigaku) was conducted in the $2 \theta$ range from $10^{\circ}$ to $80^{\circ}$. Infrared spectra were acquired using a Nicolet Avatar 360 FTIR spectrophotometer from 500 to $4000 \mathrm{~cm}^{-1}$ wavenumber. HRTEM (JEOL-2010) images were collected to characterize the structure and morphology of the samples. PL spectra and time-resolved decay curves were measured on a fluorescence spectrophotometer (Hitachi Model F-7000) equipped with a $150 \mathrm{~W}$ Xenon lamp as the excitation source. The phosphorescence quantum yields were measured by an Edinburgh FLS920 spectrophotometer with an integrating sphere. The parameters are listed as follows: a microsecond pulse lamp light source; excitation wavelength: $260 \mathrm{~nm}$; sample window: $50 \mathrm{~ms}$; excitation period: $60 \mathrm{~ms}$; delay after excitation: $0.1 \mathrm{~ms}$; step size: $1 \mathrm{~nm}$; dwell time: $0.1 \mathrm{~s}$. And then the quantum yield was calculated after measuring the excitation area and emission peak area of the background and the samples. Thermogravimetric analysis (TGA, recorded by a TG-DSC system, Netzsch) was conducted from room temperature to $1000{ }^{\circ} \mathrm{C}$ at a rate of $10^{\circ} \mathrm{C} / \mathrm{min}$ in an air atmosphere. XPS experiments were performed using a Thermo Fisher 250Xi X-ray photoelectron spectrometer with a monochromatic Al Ka X-ray source. Electron spin-resonance spectroscopy was recorded on an electron spin resonance instrument (JES FA200, JEOL, Japan).

\section{Data availability}

The data that supports the findings of this study are available from the corresponding authors upon request.

Received: 25 February 2020; Accepted: 12 October 2020;

Published online: 05 November 2020

\section{References}

1. Ma, X., Xu, C., Wang, J. \& Tian, H. Amorphous pure organic polymers for heavy-atom-free efficient room-temperature phosphorescence emission. Angew. Chem. Int. Ed. 57, 10854-10858 (2018)

2. An, Z. et al. Stabilizing triplet excited states for ultralong organic phosphorescence. Nat. Mater. 14, 685-690 (2015).

3. Yang, X. \& Yan, D. Long-afterglow metal-organic frameworks: reversible guest-induced phosphorescence tunability. Chem. Sci. 7, 4519-4526 (2016).

4. Cai, S. et al. Visible-light-excited ultralong organic phosphorescence by manipulating intermolecular interactions. Adv. Mater. 29, 1701244 (2017).

5. Li, D. et al. Amorphous metal-free room-temperature phosphorescent small molecules with multicolor photoluminescence via host-guest and dualemission strategy. J. Am. Chem. Soc. 140, 1916-1923 (2018).

6. Cai, S. et al. Enhancing ultralong organic phosphorescence by effective $\pi$-type halogen bonding. Adv. Funct. Mater. 28, 1705045 (2018).

7. He, Z. et al. White light emission from a single organic molecule with dual phosphorescence at room temperature. Nat. Commun. 8, 416 (2017).

8. Gu, L. et al. Colour-tunable ultra-long organic phosphorescence of a singlecomponent molecular crystal. Nat. Photon. 13, 406-411 (2019).

9. Cheng, Z. et al. Ultralong phosphorescence from organic ionic crystals under ambient conditions. Angew. Chem. Int. Ed. 57, 678-682 (2018).

10. Bian, L. et al. Simultaneous enhancing efficiency and lifetime of ultralong organic phosphorescence materials by molecular self-assembly. J. Am. Chem. Soc. 140, 10734-10739 (2018).

11. Lucenti, E. et al. H-aggregates granting crystallization-induced emissive behavior and ultralong phosphorescence from a pure organic molecule. J. Phys. Chem. Lett. 88, 1894-1898 (2017).

12. $0 \mathrm{w}$ ? $>$ Qu, B., Zhang, B., Wang, L., Zhou, R. \& Zeng, X. Mechanistic study of the persistent luminescence of $\mathrm{CaAl}_{2} \mathrm{O}_{4}: \mathrm{Eu}, \mathrm{Nd}$. Chem. Mater. 27, 2195-2202 (2015).

13. Zhao, W. et al. Rational molecular design for achieving persistent and efficient pure organic room-temperature phosphorescence. Chemistry 1, 592-602 (2016).
14. Wang, J. et al. A facile strategy for realizing room temperature phosphorescence and single molecule white light emission. Nat. Commun. 9, 2963 (2018)

15. Zhao, W. et al. Boosting the efficiency of organic persistent roomtemperature phosphorescence by intramolecular triplet-triplet energy transfer. Nat. Commun. 10, 1595 (2019).

16. Shen, C. et al. Bright and multicolor chemiluminescent carbon nanodots for advanced information encryption. Adv. Sci. 6, 1802331 (2019).

17. Li, W. et al. Carbon dot-silica nanoparticle composites for ultralong lifetime phosphorescence imaging in tissue and cells at room temperature. Chem. Mater. 31, 9887-9894 (2019).

18. Liu, J. et al. Carbon dots in zeolites: a new class of thermally activated delayed fluorescence materials with ultralong lifetimes. Sci. Adv. 3, el603171 (2017).

19. Li, J. Y. et al. Carbon dots-in-matrix boosting intriguing luminescence properties and applications. Small 15, 1805504 (2019).

20. Deng, Y. et al. Long lifetime pure organic phosphorescence based on water soluble carbon dots. Chem. Commun. 49, 5751 (2013).

21. Dong, X. et al. Efficient long lifetime room temperature phosphorescence of carbon dots in a potash alum matrix. J. Mater. Chem. C 3, 2798-2801 (2015).

22. Jiang, K., Wang, Y., Cai, C. \& Lin, H. Activating room temperature long afterglow of carbon dots via covalent fixation. Chem. Mater. 29, 4866-4873 (2017).

23. Tang, G. et al. One-step preparation of silica microspheres with super-stable ultralong room temperature phosphorescence. J. Mater. Chem. C 7, 8680-8687 (2019)

24. Shi, W. et al. Defect-stabilized triplet state excitons: toward ultralong organic room-temperature phosphorescence. Adv. Funct. Mater. 28, 1804961 (2018).

25. Josepha, J. \& Anappara, A. Cool white, persistent room-temperature phosphorescence in carbon dots embedded in a silica gel matrix. Phys. Chem. Chem. Phys. 19, 15137-15144 (2017).

26. Tian, Z. et al. Multilevel data encryption using thermal-treatment controlled room temperature phosphorescence of carbon dot/polyvinylalcohol composites. Adv. Sci. 5, 1800795 (2018).

27. Tan, J. et al. Large-scale synthesis of $\mathrm{N}$-doped carbon quantum dots and their phosphorescence properties in a polyurethane matrix. Nanoscale 8, 4742-4747 (2016).

28. $\mathrm{Li}, \mathrm{Q}$. et al. Induction of long-lived room temperature phosphorescence of carbon dots by water in hydrogen-bonded matrices. Nat. Commun. 9, 734 (2018).

29. Li, Q. et al. Efficient room-temperature phosphorescence from nitrogen-doped carbon dots in composite matrices. Chem. Mater. 28, 8221-8227 (2016).

30. Tao, S. et al. Design of metal-free polymer carbon dots: a new class of room temperature phosphorescent materials. Angew. Chem. Int. Ed. 57, 2393-2398 (2018).

31. Lin, C., Zhuang, Y., Li, W., Zhou, T. \& Xie, R. Blue, green, and red full-color ultralong afterglow in nitrogen-doped carbon dots. Nanoscale 11, 6584-6590 (2019).

32. Li, W. et al. A universal strategy for activating the multi-color room temperature afterglow of carbon dots in a boric acid matrix. Angew. Chem. Int. Ed. 131, 7356-7361 (2019).

33. Wang, Z., Smith, A., Wang, W. \& Sun, L. Versatile nanostructures from rice husk biomass for energy applications. Angew. Chem. Int. Ed. 57, 13722-13734 (2018).

34. Wang, Z. et al. Design and fabrication of highly photoluminescent carbon incorporated silica from rice husk biomass. Ind. Eng. Chem. Res. 58, 114688-114694 (2019).

35. Mohd, S. et al. Synthesis of silicon nanoparticles from rice husk and their use as sustainable fluorophores for white light emission. ACS Sustain. Chem. Eng. 6, 6203-6210 (2018).

36. Wang, Z. et al. Large-scale and controllable synthesis of graphene quantum dots from rice husk biomass: a comprehensive utilization strategy. ACS Appl. Mater. Interfaces 8, 1434-1439 (2016).

37. Renlund, G., Prochazka, S. \& Doremus, R. Silicon oxycarbide glasses: part II. structure and properties. J. Mater. Res. 6, 2723-2734 (1991).

38. Tan, J., Zhang, J., Zhang, L. \& Yue, D. Synthesis of amphiphilic carbon quantum dots with phosphorescence properties and their multifunctional applications. J. Mater. Chem. C 4, 10146-10153 (2016).

39. Jiang, K., Wang, Y., Cai, C. \& Lin, H. Conversion of carbon dots from fluorescence to ultralong room-temperature phosphorescence by heating for security applications. Adv. Mater. 30, 1800783 (2018).

40. Jiang, K. et al. Triple-mode emission of carbon dots: applications for advanced anti-counterfeiting. Angew. Chem. Int. Ed. 55, 7231-7235 (2018).

41. Tan, J., Ye, Y., Ren, X., Zhao, W. \& Yue, D. High pH-induced efficient roomtemperature phosphorescence from carbon dots in hydrogen-bonded matrices. J. Mater. Chem. C 6, 7890-7895 (2018).

42. Long, P. et al. Self-protective room-temperature phosphorescence of fluorine and nitrogen co-doped carbon dots. Adv. Funct. Mater. 28, 1800791 (2018). 
43. Xu, S., Chen, R., Zheng, C. \& Huang, W. Excited state modulation for organic afterglow: materials and applications. Adv. Mater. 28, 9920-9940 (2016).

44. Burner, Pauline et al. Evidence of organic luminescent centers in sol-gelsynthesized yttrium aluminum borate matrix leading to bright visible emission. Angew. Chem. Int. Ed. 56, 13995-13998 (2017).

45. Wei, K. et al. Temperature-dependent excitonic photoluminescence excited by two-photon absorption in perovskite $\mathrm{CsPbBr} 3$ quantum dots. Opt. Lett. 16, 3821-3824 (2016).

46. Oliinyk, B. et al. Are fluorescent silicon nanoparticles formed in a one-pot aqueous synthesis? Chem. Mater. 31, 7167-7172 (2019).

47. Zhao, L. et al. Room-temperature metal-activator-free phosphorescence from mesoporous silica. Phys. Chem. Chem. Phys. 13, 2387-2393 (2011).

48. Wei, J. et al. Induction of strong long-lived room-temperature phosphorescence of N-phenyl-2-naphthylamine molecules by confinement in a crystalline dibromobiphenyl matrix. Angew. Chem. Int. Ed. 55, 15589-15593 (2016).

49. Green, D. et al. Controlling the fluorescence and room-temperature phosphorescence behaviour of carbon nanodots with inorganic crystalline nanocomposites. Nat. Commun. 10, 206 (2019).

50. Gao, R., Mei, X., Yan, D., Liang, R. \& Wei, M. Nano-photosensitizer based on layered double hydroxide and isophthalic acid for singlet oxygenation and photodynamic therapy. Nat. Commun. 9, 2798 (2018).

51. Cai, S. et al. Enabling long-lived organic room temperature phosphorescence in polymers by subunit interlocking. Nat. Commun. 10, 4247 (2019).

\section{Acknowledgements}

M.Z. and Y.L. thank the support by the National Natural Science Foundation of China (21571066, U1501242, and 21571067). M.Z. also thanks the support from the China Scholarship Council (CSC) for conducting 1 year of visiting research at the University of Connecticut.

\section{Author contributions}

L.S., Y.L., and M.Z. conceived the idea and supervised the research. Y.S. and S.L. synthesized samples and performed the characterizations. Y.S., Y.L., and M.Z. carried out characterizations and analyzed the data. S.W., X.P., G.H., and C.H. conducted structure characterization. A.T.S. and W.W. analyzed the data and provided important suggestions on phosphorescence mechanism. L.S., S.Z., and M.Z. developed the applications. Y.S., L.S., and M.Z. wrote the first draft of the paper, and all authors contributed to revise the paper.

\section{Competing interests}

The authors declare no competing interests.

\section{Additional information}

Supplementary information is available for this paper at https://doi.org/10.1038/s41467 020-19422-4.

Correspondence and requests for materials should be addressed to L.S., Y.L. or M.Z.

Peer review information Nature Communications thanks the anonymous reviewer(s) for their contribution to the peer review of this work.

Reprints and permission information is available at http://www.nature.com/reprints

Publisher's note Springer Nature remains neutral with regard to jurisdictional claims in published maps and institutional affiliations.

cC Open Access This article is licensed under a Creative Commons Attribution 4.0 International License, which permits use, sharing, adaptation, distribution and reproduction in any medium or format, as long as you give appropriate credit to the original author(s) and the source, provide a link to the Creative Commons license, and indicate if changes were made. The images or other third party material in this article are included in the article's Creative Commons license, unless indicated otherwise in a credit line to the material. If material is not included in the article's Creative Commons license and your intended use is not permitted by statutory regulation or exceeds the permitted use, you will need to obtain permission directly from the copyright holder. To view a copy of this license, visit http://creativecommons.org/ licenses/by/4.0/.

(C) The Author(s) 2020 